\title{
Aplicación del coeficiente de Gini y la semivarianza como estimadores del riesgo en la selección de proyectos
}

INDUSTRIAL ENGINEERING

\section{Application of Gini coefficient and semivariance as estimators of risk in project selection}

\author{
Diego F. Manotas-Duque ${ }^{\S}$, Alejandro Estrada-Bedón**, José A. Uribe-Rodríguez*** \\ *Escuela de Ingeniería Industrial y Estadística Universidad del Valle, Cali, Colombia \\ sdiego.manotas@correounivalle.edu.co \\ **Servicio de Cooperación Transferencia de Tecnología, Centro de Investigación de Caña de Azúcar de Colombia, \\ aestrada@cenicana.org \\ ***Facultad de Ciencias Económicas, Universidad de San Buenaventura, Cali, Colombia \\ jaurodri@usbcali.edu.co
}

(Recibido: Septiembre 9 de 2010- Aceptado: 27 de 2011)

\begin{abstract}
Resumen
La presente investigación tiene sus orígenes en la creciente necesidad de conocer y aplicar metodologías de selección y ordenamiento de proyectos, enmarcadas dentro de la utilización eficiente y efectiva de los diferentes recursos productivos de las organizaciones, que por ser escasos, deben utilizarse de la mejor manera posible. Lo anterior llevó al diseño y desarrollo de una herramienta de optimización que permite planear y ordenar un conjunto de proyectos dentro de un plan de inversión, de tal forma que se maximice el beneficio total generado por su ejecución. Por lo cual, se muestra de forma detallada la formulación de dos modelos matemáticos teóricos (Media-Gini y Media-Semivarianza) utilizados para hallar la solución de la problemática, es decir la obtención de un portafolio óptimo. El primer modelo involucra en su función objetivo tres índices de valoración: económico, financiero y social, y utiliza el coeficiente de Gini como estimador del riesgo del portafolio, el segundo modelo tiene una función bi-objetivo, que consiste en maximizar el valor presente neto (VPN) del portafolio y la minimización del riesgo de éste, los cuales normalmente se encuentran en conflicto, ya que la optimización de uno usualmente va en detrimento del otro, el estimador del riesgo en este modelo es la semivarianza. Con el objetivo de validar los modelos y su explicación metodológica, estos fueron aplicados a un caso particular adaptado de una empresa de servicios públicos de la región, teniendo como base la información suministrada por ésta, en cuanto a disponibilidad de capital, mano de obra y maquinaria, así mismo sus expectativas en torno a el riesgo máximo permitido y la rentabilidad mínima esperada.
\end{abstract}

Palabras Clave: Priorización proyectos, Media-Gini, Media-semivarianza, Riesgo, Rentabilidad, Optimización.

\begin{abstract}
This research has its origins in the growing need to understand and apply methodologies for project selection and ordering, framed within the efficient and effective utilization of productive resources of different organizations, which, being scarce, must be used in the best possible way. This led to the design and development of an optimization tool that allows to plan and manage a set of projects within an investment plan, so as to maximize the total profit generated by its implementation. Therefore, shown in detail the mathematical formulation of two theoretical models (Mean-Gini and Media-semivariance) used for the solution of the problem, obtaining an optimal portfolio. The first model in its objective function involves three levels of valuation: economic, financial and social, and uses the Gini coefficient as an estimator of portfolio risk, the second model is a bi-objective function, which is to maximize the net present value (VPN) portfolio and minimizing the risk of it, which usually are in conflict, as an optimization to the detriment of another, the estimate of risk in this model is the semivariance. With the aim of validating the models and methodological explanation, these were applied to a particular case adapted from a utility company in the region, on the basis of information provided by it, in terms of availability of capital, labor and machinery, and the same expectations about the maximum risk and the minimum return expected.
\end{abstract}

Keywords: Prioritizing projects, Media-Gini, Media-semivariance, Risk, Performance, Optimization. 


\section{Introducción}

La creciente necesidad que tienen las organizaciones de tomar decisiones más acertadas acerca de sus prioridades en los diferentes procesos que ellas desarrollan, hace relevante la identificación de aquellas actividades que agregan valor. Por este motivo, es imperioso que se desarrollen elementos de carácter conceptual y conductual que permitan a las organizaciones tomar decisiones acordes con sus políticas y planes. Sin importar el tipo de organización (pública o privada), la asignación adecuada de los recursos disponibles a los diferentes proyectos de inversión, se constituye en una tarea que requiere un mayor análisis.

Sefair \& Medaglia, (2008) consideran que: al mecanismo de selección y ordenamiento de proyectos puede llegar a ser complejo, cuando la organización cuenta con múltiples recursos para asignarse, múltiples criterios de evaluación para sus proyectos, restricciones técnicas y un número considerable de alternativas. La complejidad de este proceso hace necesario el desarrollo de nuevas herramientas matemáticas y computacionales.

Existe una extensa literatura sobre el tema, en la cual es posible destacar algunos trabajos que proponen soluciones alternativas ante la complejidad del problema de selección estudiado. Es el caso de Ferrnández \& Gomez. (2007), quienes aplicaron una heurística basada en redes neuronales con el fin de encontrar la frontera eficiente asociada con el problema de mediavarianza, comparándola con los resultados obtenidos por medio de algoritmos genéticos, búsqueda tabú y temple simulado; concluyendo que ninguna de las cuatro metaheurísticas es superior a las otras para resolver el problema de la selección del portafolio. En la misma línea, Branke et al. (2008), proponen un nuevo algoritmo evolutivo multi-objetivo, el cual, según los autores, encuentra en un menor tiempo la frontera eficiente, frente a otros algoritmos de este tipo desarrollados anteriormente.

Medaglia et al. (2008) propusieron un modelo de programación binaria bi-objetivo cuya solución se obtuvo mediante el método de $\varepsilon$-restricciones; de igual forma, utilizando este tipo de herramienta, programación binaria, Marantes et al. (2005) y Sitaram et al. (2004) propusieron un método de solución basado en algoritmos genéticos para explorar eficientemente el conjunto de soluciones factibles, cuyos componentes son binarios.

Medaglia et al. (2007) proponen un algoritmo evolutivo multiobjetivo basado en una articulación posterior de preferencias, y en la teoría de optimización lineal permitiendo realizar una aproximación a la frontera eficiente compuesta por soluciones no dominantes estocásticamente. Este nuevo método evalúa proyectos parcialmente iniciados, objetivos múltiples, interdependencias de los proyectos (en los objetivos), y una estructura lineal para las restricciones de recursos. Se incluyen elementos inciertos en los objetivos para modelarse como coeficientes aleatorios en expresiones complejas no lineales. Inicialmente, el método simula el proyecto y evalúa el comportamiento de las mediciones (objetivos) sujeto a los elementos aleatorios.

Ghorbani \& Rabbani (2009) proponen una meta heurística multi-objetivo para el problema de selección de proyectos, la cual considera dos funciones objetivo, la maximización del beneficio total esperado y la minimización de la sumatoria de la desviación estándar del recurso localizado entre cada periodo de tiempo sucesivo. El algoritmo empleado para construir las soluciones no dominantes es una meta heurística basada en un algoritmo mimético, este se utiliza para obtener diversas soluciones no dominantes con alta calidad. Estos autores proponen la incorporación del criterio de la minimización de la sumatoria de la variación absoluta de la asignación de recursos entre períodos sucesivos de tiempo, que según la literatura no ha sido considerada.

Huang (2007a) en su estudio realiza una introducción sobre la teoría en relación a las variables difusas y las variables aleatorias difusas para abordar sus modelos. En los modelos desarrollados se propone como estrategia de solución, un algoritmo híbrido inteligente y un algoritmo genético. La justificación de la utilización de todas estas técnicas está ligada a la complejidad que se encuentra en la modelación de 
los retornos de la inversión al considerar que los algoritmos tradicionales son insuficientes para encontrar una solución óptima. A pesar de considerar que los algoritmos propuestos son adecuados, encuentra un inconveniente en el tiempo de ejecución de los algoritmos, utilizando las variables aleatorias difusas, puesto que gran parte de este tiempo se consume en la simulación de estas variables. Huang (2007b) propone un mejoramiento de su anterior trabajo, sin embargo, llega a la misma conclusión que en su trabajo anterior. Huang (2008) utiliza este mismo algoritmo pero construye una nueva definición de riesgo que considera es más indicada para utilizarse en ambientes difusos y analiza un nuevo criterio para la clasificación jerárquica del riesgo. A pesar de que estas modificaciones son muy apropiadas para la solución de la problemática, el tiempo de ejecución sigue siendo el gran inconveniente de sus modelos.

Lin et al. (2005) proponen un acercamiento sistemático incorporando lógica difusa junto con las matrices asociadas al portafolio de proyectos, con el objetivo de ayudar a los gerentes en la toma de decisiones para que el entendimiento de ellos sobre el tema sea amigable y pueda revelar de una mejor forma la bondad global que les ofrece su portafolio. En su modelo utilizan la programación lineal entera como método de solución, ya que consideran que es un método cuantitativo eficaz en la lógica de asignación óptima de recursos escasos y además permite describir con una mayor confiabilidad las ponderaciones del problema de incertidumbre.

Tiryaki \& Ahlatcioglu (2009) presentan un trabajo con el propósito de incorporar la teoría difusa al AHP con el fin de aplicarlo al problema de selección del portafolio de inversiones. En su trabajo aplican el método utilizado por Enea \& Piazza (2004) y el RCFAHP (Revised Constrained Fuzzy AHP), para aplicarlo al problema de seleccionar acciones en la bolsa de Estambul. Si bien los dos métodos proporcionan buena información sobre la clasificación jerárquica, consideran más apropiado el método RCFAHP, puesto que modela de una mejor forma los ambientes inciertos. La metodología AHP se utilizó para calcular la importancia de los factores y de esta forma conocer el peso dentro de la función objetivo del modelo de programación lineal que se construyó posteriormente, decidiéndose que proyecto realizar.

Hasta el momento no existe un modelo que sea capaz de satisfacer todas las necesidades en la selección y priorización de un portafolio de forma práctica, por tal motivo, Ringuest et al. (2004) presentan un modelo, adaptado de la literatura sobre optimización del portafolio de proyectos de inversión bajo riesgo, que proporciona medios prácticos para facilitar la toma de decisiones, puesto que consideran que los primeros trabajos sobre el tema no manejan en forma adecuada la modelación del riesgo y otros no proporcionan resultados eficaces. El método que proponen lo consideran simple y muy intuitivo, ya que requiere sólo de dos parámetros, el retorno esperado y el coeficiente de Gini. Este último reemplaza la varianza del método de Markowitz. Con estos dos parámetros también se permite la determinación relativamente simple de la dominación estocástica. Los resultados obtenidos son bastante atractivos, sobre todo porque los encargados de la toma de decisiones, tendrían una herramienta útil y fácil de manejar, sin embargo en el modelo sólo se tiene una restricción, que es la asociada al retorno esperado, pero se sabe que en la realidad pueden existir muchas, y es allí, donde pueden surgir los inconvenientes y donde se pondría a prueba el modelo. De todas formas los autores aseguran que la aparición de nuevas restricciones, no supone mayores problemas.

Medaglia et al. (2008) formularon un modelo de tipo lineal entero-mixto multiobjetivo que maximiza la suma de pesos de los valores presentes netos económicos $\mathrm{y}$ financieros normalizados y un índice de impacto social. Simultáneamente, se debe generar la satisfacción de un conjunto de relaciones de precedencia sobre los proyectos, inicio temprano y tardío del proyecto, limites de inversión exógena y generación de flujos de caja endógenos. La formulación incluye un conjunto candidato de proyectos, el número mínimo o máximo de proyectos a ser incluido en el portafolio y un conjunto de relaciones de precedencia entre los proyectos, permitiendo de esta forma identificar que proyectos seleccionar y la fecha de inicio de cada proyecto. 


\section{Metodología}

La obtención de un portafolio de inversión presenta características generales aplicables a cualquier rama de la actividad económica. Sin embargo, cada una de ellas presenta características particulares, que deben ser consideradas por aquellos que tienen la responsabilidad de analizar y evaluar los proyectos para formar el portafolio. El proceso de inversión en un portafolio también implica la toma de decisiones sobre tres preguntas fundamentales: ¿en cuáles proyectos invertir?, ¿cuánto es el capital que se debe asignar a cada uno de ellos? y ¿en qué momento hacerlo? Para dar respuestas a estos interrogantes, es necesario realizar un procedimiento que está constituido por cinco etapas básicas: delimitación de los objetivos de inversión, determinación de la política de inversión, selección de la estrategia de conformación del portafolio, selección de los proyectos y finalmente la medición y evaluación del desempeño. En el presente documento no se tiene en cuenta la selección de la estrategia para la conformación de portafolios, ya que su aplicación está dirigida al mercado de valores, tampoco la medición y evaluación del desempeño, pues esto supone el seguimiento de los resultados obtenidos en un contexto real.

Para dar cumplimiento a las dos primeras etapas de dicho proceso, se necesita la información entregada por los inversionistas (preferencias, limitaciones, prioridades, historia, etc.). Como se mencionó antes, en el caso particular de esta investigación, el inversionista está representado por una empresa de servicios públicos de la región. Esta información se encuentra en la sección 2.8 y se constituye en la base para generar el modelo computacional.

\subsection{Construcción del modelo teórico}

De manera particular, para la formulación de los modelos de optimización utilizados en la presente investigación, se tuvo en cuenta el contexto general, es decir, la primera etapa se enfoca en la revisión y estudio de teorías, conceptos y modelos de optimización, alusivos al tema de selección y priorización de planes de inversión. Con base en esto y con la información suministrada por el inversionista fue posible identificar modelos que pueden ser adaptados para resolver el problema general y ser aplicados a un caso particular. La Figura 1 corresponde al proceso general de la construcción de un modelo teórico.

\subsection{Modelos Propuestos}

Partiendo del modelo de media-varianza planteado por Markowitz (1952) para la selección de proyectos de inversión bajo riesgo, la presente investigación centra su interés en un aspecto fundamental, que es la inserción de dos indicadores (social y financiero) en la función objetivo del primer modelo, los cuales normalmente no se tienen en cuenta para la resolución de problemas de selección de portafolios de inversión.

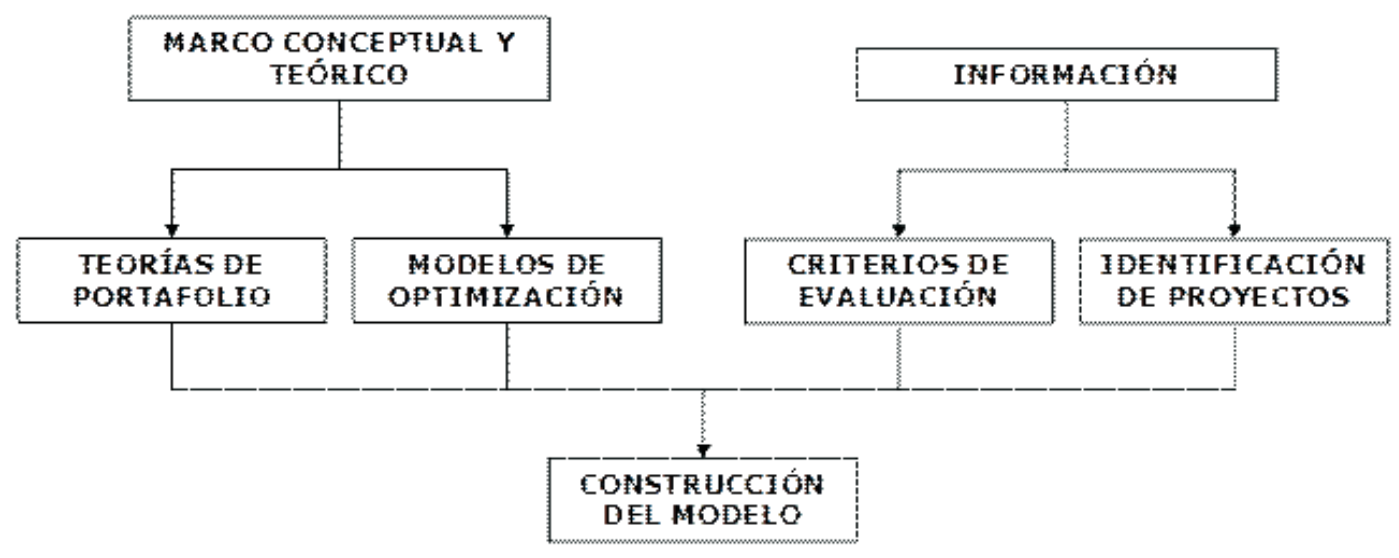

Figura 1. Esquema del proceso general de construcción de un modelo teórico 
Sin embargo, estos resultan relevantes en la obtención de un portafolio óptimo, situación que se describirá posteriormente de una forma más detallada. Para cuantificar el alcance de este modelo se comparó con otra propuesta de solución de esta problemática, en la cual no se consideraban estos indicadores. Para la resolución de ambos modelos se utilizó el programa de análisis de riesgo y pronóstico Crystal Ball ${ }^{\circledR}$ y el aplicativo Optquest contenido en el mismo.

El primer modelo (Media-Gini) corresponde a un caso de programación lineal entera mixta, el cual incluye en la función objetivo los diferentes indicadores: económico, financiero y social; mientras que el segundo (Media-Semivarianza) obedece a un caso bi-objetivo, que supone dos objetivos que además de ser conflictivos, uno de ellos es cuadrático y requiere ser llevado a forma lineal. En el primero de ellos se utiliza el coeficiente de Gini como medida de riesgo, mientras que en el segundo el indicador del riesgo es la semivarianza.

\subsection{Modelo Media-Gini (MG)}

La función objetivo busca maximizar el impacto de los proyectos realizados sobre la empresa y obedece a un caso de programación lineal entera mixta, por cuanto las variables de decisión $\left(y_{i t}\right)$ son binarias, el parámetro $\lambda_{\text {kit }}$ es adimencional, lo mismo que los índices $l_{\text {kit }}$ su formulación se muestra en la Ec. (1):

$$
\operatorname{Max} \quad Z=\sum_{\mathrm{K}=1}^{\mathrm{r}} \sum_{\mathrm{i}=1}^{\mathrm{n}} \sum_{\mathrm{t}=1}^{\mathrm{t} \max } \lambda_{\mathrm{kit}} \mathrm{l}_{\mathrm{kit}} y_{\mathrm{it}}
$$

$1_{\text {kit }}$ : es el índice $k$ del proyecto $i$ en el periodo $t$ a ser evaluado, $\mathrm{i}=1,2,3, \ldots, \mathrm{n}, \mathrm{k}=1,2, \ldots, \mathrm{r}, \mathrm{t}=0,1, . ., \mathrm{tmax}$ , para esta investigación se han considerado tres índices (económico, financiero y social) y cuatro años para el horizonte de tiempo.

$\lambda_{\text {kit }}$ : es el peso para el índice $\mathrm{k}$ del proyecto $\mathrm{i}$, en el periodo $t$, este peso es dado a priori y en este trabajo fue obtenido mediante AHP (Analytical Hierarchy Process), de tal forma que para cada periodo y cada índice se debe satisfacer la Ec. (2).

$$
0 \leq \lambda_{\text {kit }} \leq 1 ; \quad \text { con } \quad \sum_{\text {i=1 }}^{n} \lambda_{\text {kit }}=1
$$

Las demás restricciones se ilustran a continuación:

$$
\mathcal{G}=2 \operatorname{Cov}\left[\sum_{\mathrm{i}=1}^{\mathrm{n}} \sum_{\mathrm{t}=1}^{\operatorname{tmax}} \beta_{\mathrm{it}} \mathrm{R}_{\mathrm{it}} y_{\mathrm{it}}, \mathrm{F}\left(\sum_{\mathrm{i}=1}^{\mathrm{n}} \sum_{\mathrm{t}=1}^{\operatorname{tmax}} \beta_{\mathrm{it}} \mathrm{R}_{\mathrm{it}} y_{\mathrm{it}}\right)\right] \leq \rho
$$

$\mathcal{G}$ : es el coeficiente de Gini y corresponde a la estimación del riesgo del portafolio. Su cálculo se halló teniendo como referencia los trabajos realizados por Shalit \& Yitzhaki(1984), donde F representa la función de distribución de probabilidad acumulada; $\mathrm{R}_{\mathrm{it}}$ : representa el índice de rentabilidad del proyecto $i$ en el periodo $t y$ es el nivel de riesgo deseado del portafolio, es decir, el máximo riesgo que puede soportar el inversionista.

$$
\sum_{i=1}^{n} \sum_{t=1}^{T} \beta_{i t} y_{i t} \leq 1
$$

$\beta_{\text {it }}$ : Es la proporción de capital utilizado por el proyecto i $\mathrm{C} \mathrm{P}$ en el periodo $\mathrm{t}$, del dinero disponible total en ese periodo.

$$
\mathrm{k}_{\mathrm{l}} \leq \sum_{\mathrm{i}=1}^{\mathrm{n}} \sum_{\mathrm{t}=1}^{\mathrm{tmax}} y_{\mathrm{it}} \leq \mathrm{k}_{\mathrm{u}}
$$

$\mathrm{K}_{1} \mathrm{y} \mathrm{K}_{\mathrm{u}}$ : Representan respectivamente el mínimo y el máximo número de proyectos, para ser incluidos en el portafolio.

$$
\sum_{i=1}^{n} \beta_{i} \bar{R}_{i} y_{i} \geq \gamma
$$

$\gamma$ : Es la rentabilidad mínima esperada por el inversionista, está dada en términos porcentuales. 
$\beta_{\mathrm{i}}$ : Es la proporción total de capital utilizado por el proyecto $\mathrm{i}$

$\overline{\mathrm{R}}_{\mathrm{i}}$ : Es el índice promedio de rentabilidad del proyecto $i$

$$
\sum_{\mathrm{i}=1}^{\mathrm{n}} \sum_{\mathrm{t}=1}^{\mathrm{tmax}} \mathrm{H}_{\mathrm{it}} y_{\mathrm{it}} \leq \mathrm{O}_{\mathrm{t}}
$$

$\mathrm{H}_{\mathrm{it}}$ : Es el número de horas-hombre necesarias para realizar el proyecto $\mathrm{i} \epsilon \mathrm{P}$ en el periodo $t$

$\mathrm{O}_{\mathrm{t}}$ : Es el número de horas-hombre disponibles para el periodo $t$

$$
\sum_{\mathrm{i}=1}^{\mathrm{n}} \sum_{\mathrm{t}=1}^{\mathrm{tmax}} \mathrm{Q}_{\mathrm{it}} y_{\mathrm{it}} \leq \mathrm{M}_{\mathrm{t}}
$$

$\mathrm{M}_{\mathrm{t}}$ : Es el número de horas-máquina disponibles para el periodo $t$

$\mathrm{Q}_{\mathrm{it}}$ : Es el número de horas-máquina necesarias para realizar el proyecto $i$ en el periodo $t$

El hecho de seleccionar el proyecto i para formar parte del portafolio óptimo, lleva consigo la necesidad de activar la variable de decisión auxiliar correspondiente a cada periodo, esto se logra mediante

$$
y_{i t}=X_{i}
$$

$\mathrm{RP}=\{(\mathrm{i}, \mathrm{j})\}$ es un conjunto formado por la relación de precedencia entre el proyecto i y el proyecto $j$, es decir, el hecho de incluir el proyecto $i$ en el portafolio óptimo, puede llevar consigo la obligación de incluir también el proyecto j en éste, esta situación conlleva a tener dos tipos de restricción de precedencia presentados en las Ec. $10 \mathrm{y} 11$.

Cuando el proyecto j sólo pueda tenerse en cuenta para su evaluación, una vez que haya sido seleccionado el proyecto i, se deben formular las siguientes restricciones de precedencia

$$
y_{j t} \leq y_{i t} \quad(\mathrm{i}, \mathrm{j}) \in \mathrm{RP}
$$

Cuando el proyecto j sólo dependa de la inclusión o no, en el portafolio óptimo el proyecto $i$, se deben formular las siguientes restricciones de precedencia.

$$
y_{j t}=y_{i t} \quad(\mathrm{i}, \mathrm{j}) \in \mathrm{RP}
$$

Restricciones obvias

$$
y_{i t}, x_{i t} \in\{0,1\}
$$

El cálculo de valores para los índices 1 se realiza de la siguiente forma:

Índice Económico (Rentabilidad)

$$
\mathrm{R}_{\mathrm{it}}=\frac{\mathrm{VPN}_{\mathrm{i}(\mathrm{t})}-\mathrm{VPN}_{\mathrm{i}(\mathrm{t}-1)}}{\mathrm{VPN}_{\mathrm{i}(\mathrm{t}-1)}}
$$

La rentabilidad promedio del proyecto $i$

$$
\overline{\mathrm{R}}_{\mathrm{it}}=\frac{1}{\operatorname{tmax}} \sum_{\mathrm{t}=1}^{\mathrm{tmax}} \mathrm{R}_{\mathrm{it}}
$$

El índice financiero

$$
\mathrm{ME}_{\mathrm{it}}=\frac{\mathrm{EB}_{\mathrm{it}}}{\mathrm{ING}_{\mathrm{it}}}
$$

Donde: MEit: Es el margen Ebitda del proyecto i en periodo t; $\mathrm{ING}_{\mathrm{it}}$ : Es el ingreso del proyecto i en el periodo t,EBit : Es el ingreso EBITDA del proyecto $i$ en el periodo $t$

El índice Social

$$
\mathrm{S}_{\mathrm{it}}=\frac{\mathrm{Pb} \mathrm{b}_{\mathrm{it}}}{\mathrm{N}}
$$

$\mathrm{S}_{\mathrm{it}}$ : Porcentaje de población beneficiada

$\mathrm{Pb}_{\mathrm{it}}$ : Es el número de personas que se benefician por la realización del proyecto i en el periodo t, para el caso se ha considerado que este valor es constante para todo el horizonte de tiempo. 
$\mathrm{N}$ : Es la población, y también se considera que su valor es constante para todo el horizonte de tiempo

\subsection{Modelo Media-Semivarianza (MS)}

La información suministrada para la elaboración del modelo MG, se constituye básicamente en la misma que se utilizó para la formulación del modelo MS, esto significa que la estructura contemplada desde la Ec. 4 hasta la Ec. 12 es común para ambos modelos. En consecuencia, este modelo está constituido por las mismas ecuaciones y la función objetivo, ya que no se incorporan los índices como ponderaciones para la toma de decisiones, y en su lugar los elementos relevantes para formar el portafolio óptimo, están representados por el VPN y el riesgo. Este último será estimado mediante la semivarianza.

Se hace también necesario utilizar una variable auxiliar $Z_{i j t}$, para asegurar la transformación lineal de la Ec. (17) y que corresponde al producto de las variables binarias $y_{i t} \mathrm{y} y_{j t}$ en el periodo t, (aunque del anterior modelo se sabe que $y_{i t}$ es igual para todo el horizonte de tiempo). Esta variable auxiliar se define así:

$$
z_{i j t}=y_{j t} * y_{i t} \quad z_{i j t} \in\{0,1\}
$$

La función objetivo de este problema busca maximizar el retorno de la inversión valorado por el valor presente neto de las inversiones (VPN) y minimizar el riesgo del portafolio que resulta de la combinación lineal de los diferentes proyectos, $\mathrm{y}$ se estima en términos de la covarianza entres los proyectos que forman parte del portafolio.

Esta función obedece a un caso de programación multi-objetivo, más específicamente bi-objetivo, donde éstos dos objetivos son conflictivos entre sí, ya que como se sabe, normalmente los proyectos altamente rentables, están asociados a niveles de riesgo también significativamente altos. La Ec. (19) corresponde a la minimización del riesgo y el cálculo se realizó con base en el trabajo realizado por Estrada (2003) y utilizado por Medaglia et al. (2008). La formulación de la función objetivo se presenta a continuación:

$$
\operatorname{Max}=\sum_{\mathrm{i}=1}^{\mathrm{n}} \sum_{\mathrm{t}=1}^{\mathrm{T}} \mathrm{E}\left(\mathrm{VPN}_{\mathrm{i}}\right) y_{\mathrm{it}}
$$

$$
\operatorname{Min}=\sum_{i \in p} \sum_{j \in p} \sum_{t=1}^{T} \operatorname{Cov}\left(\operatorname{VPN}_{i}, V_{P N}\right) Z_{i j t}
$$

Sujeto a las restricciones del modelo MG desde la Ec.4 hasta la Ec. 12.

\subsection{Modelo computacional}

En la Figura 2 se puede observar que existen tres aspectos básicos para generar un modelo computacional de simulación: la información probabilística, la información determinística y el modelo teórico. En el caso particular de esta investigación, el tercer aspecto ya está resuelto. Sin embargo, los otros dos aspectos que tienen el mismo grado de importancia, aún no han sido elaborados, por tanto, en las siguientes secciones se describirá el proceso desarrollado para la obtención de ellos.

\subsection{Información Probabilística}

Como ya se ha dicho, los tres aspectos fundamentales que componen la implementación de un modelo de simulación tienen el mismo nivel de importancia, es decir, un error en cualquiera de ellos, causará que los objetivos propuestos no sean alcanzados, sin embargo, la obtención de la información estocástica, es la que tiene una mayor probabilidad de error, ya que en todos los pasos que se deben seguir está latente la posibilidad de incurrir en él, por lo tanto esta etapa de la investigación debe tratarse con sumo cuidado.

En términos generales para la obtención de la información probabilística se deben tener en cuenta los siguientes elementos:

- Identificar claramente las variables que tienen un comportamiento aleatorio.

- Recurrir a la información histórica sobre la variable.

- Aplicar una prueba de bondad de ajuste, (Chi cuadrado, Anderson-Darling o Kolmogorov Smirnov) 


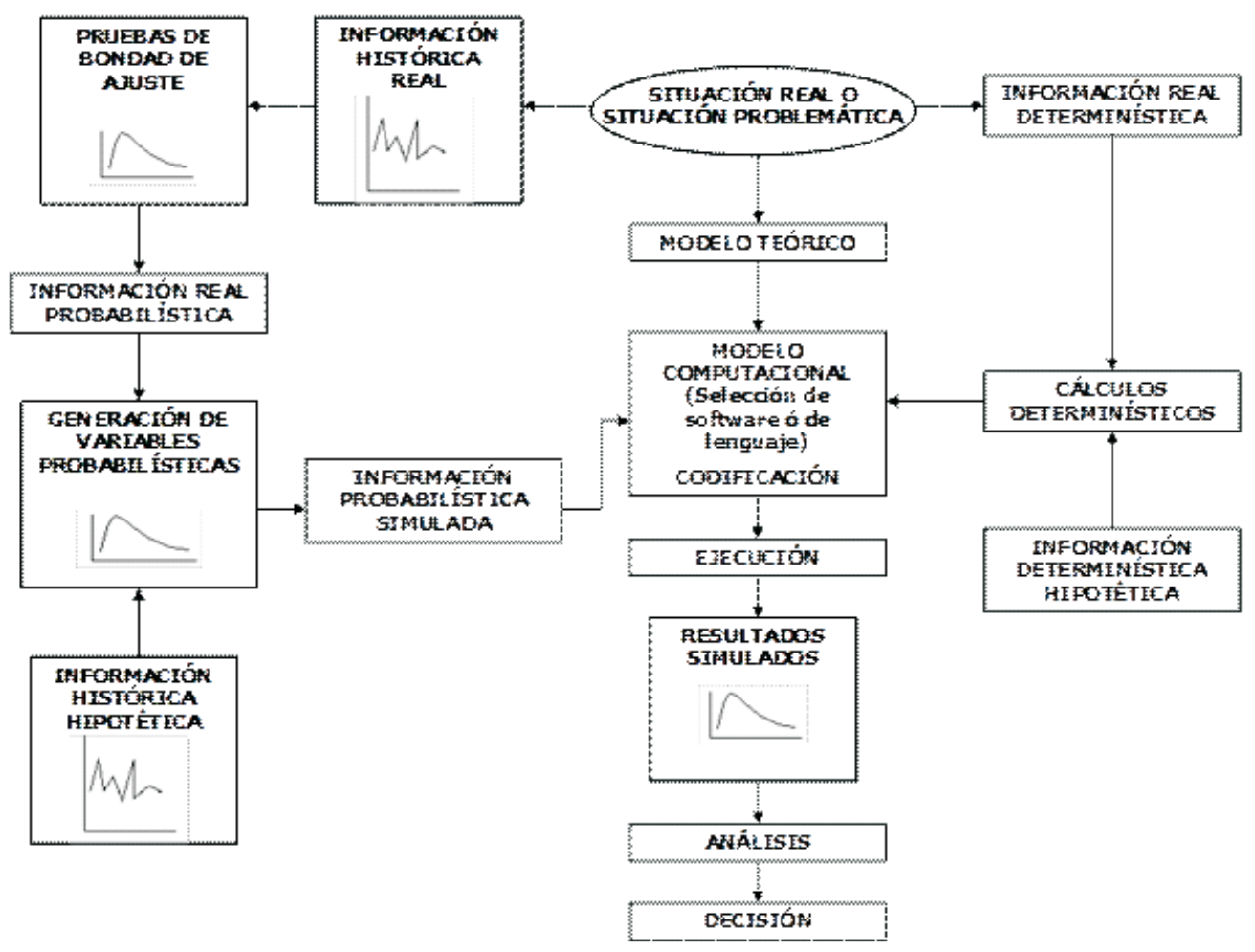

Figura 2. Esquema del proceso general de construcción del computacional y su simulación

- Crear modelos que representen el comportamiento de estas variables.

- Ingresar la información al modelo.

Para el caso del presente documento, se identificaron las variables que tenían un comportamiento aleatorio, estas son tres y corresponden al incremento en los ingresos, el margen EBITDA y el incremento en el capital de trabajo. Como no existía historia que pudiera esclarecer las distribuciones de probabilidad asociadas a ellas, se recurrió a información suministrada por un grupo de analistas expertos. Esta información se encuentra condensada en la Tabla 1, y el tipo de distribución que se asignó a todas las variables, fue la distribución triangular, basada en tres parámetros: el valor mínimo a, el valor más probable b, y el valor máximo c. Existen trabajos que sustenta el uso y las bondades de la distribución triangular, uno de ellos se puede observar en Law et al. (2000).

\subsection{Información Determinística}

De la misma forma que con la información probabilística existen pasos lógicos y secuenciales que se deben seguir, para alcanzar el objetivo. Este proceso se describe a continuación.

- Identificar claramente todos los parámetros que intervienen en el proceso

- Recurrir a la información determinística real disponible

- Realizar los cálculos pertinentes

- Ingresar la información al modelo.

En el caso particular se identificaron todos los parámetros que intervienen en el proceso, muchos de los cuales como: tasa de impuestos, disponibilidad de horas hombre y demanda de horas maquina, costo de capital, entre otros, se encuentran descritos en la sección 2.8.

Para la implementación del modelo MG 
Tabla 1. Parámetros de las variables de entrada

\begin{tabular}{|c|c|c|c|c|}
\hline Proyecto & $\begin{array}{c}\text { Parámetros de } \\
\text { la distribución } \\
\text { triangular }\end{array}$ & $\begin{array}{c}\text { Tasa de } \\
\text { crecimiento } \\
\text { de los } \\
\text { ingresos, \% }\end{array}$ & $\begin{array}{c}\text { Margen } \\
\text { EBITDA,\% }\end{array}$ & $\begin{array}{c}\text { Incremento } \\
\text { en el capital } \\
\text { de trabajo, \% }\end{array}$ \\
\hline \multirow{3}{*}{1} & Mínimo & 8 & 40 & 6 \\
\hline & Más probable & 10 & 42 & 10 \\
\hline & Máximo & 11 & 45 & 12 \\
\hline \multirow{3}{*}{2} & Mínimo & 9 & 38 & 9 \\
\hline & Más probable & 10 & 40 & 12 \\
\hline & Máximo & 13 & 42 & 13 \\
\hline \multirow{3}{*}{3} & Mínimo & 8 & 47 & 13 \\
\hline & Más probable & 9 & 48 & 15 \\
\hline & Máximo & 13 & 53 & 19 \\
\hline \multirow{3}{*}{4} & Mínimo & 10 & 45 & 15 \\
\hline & Más probable & 12 & 50 & 17 \\
\hline & Máximo & 14 & 52 & 18 \\
\hline \multirow{3}{*}{5} & Mínimo & 17 & 37 & 9 \\
\hline & Más probable & 18 & 40 & 10 \\
\hline & Máximo & 19 & 43 & 11 \\
\hline \multirow{3}{*}{6} & Mínimo & 10 & 31 & 7 \\
\hline & Más probable & 11 & 32 & 8 \\
\hline & Máximo & 12 & 33 & 10 \\
\hline \multirow{3}{*}{7} & Mínimo & 8 & 44 & 4 \\
\hline & Más probable & 9 & 46 & 5 \\
\hline & Máximo & 13 & 47 & 6 \\
\hline \multirow{3}{*}{8} & Mínimo & 10 & 43 & 4 \\
\hline & Más probable & 13 & 47 & 7 \\
\hline & Máximo & 15 & 49 & 9 \\
\hline \multirow{3}{*}{9} & Mínimo & 8 & 41 & 3 \\
\hline & Más probable & 10 & 43 & 6 \\
\hline & Máximo & 12 & 45 & 7 \\
\hline \multirow{3}{*}{10} & Mínimo & 4 & 33 & 8 \\
\hline & Más probable & 6 & 35 & 10 \\
\hline & Máximo & 8 & 37 & 14 \\
\hline
\end{tabular}

propuesto, era necesaria la obtención de las ponderaciones de los índices que se evalúan. Los cálculos de éstos, demandan un trabajo significativo y fueron obtenidos por medio del AHP (Analytical Hierarchy Process), en las Tablas 2, 3 y 4 se muestra la matriz de comparaciones pareadas, la matriz normalizada, el vector de ponderación, respectivamente, correspondiente al indicador económico.

Es importante anotar que la información para su cálculo es hipotética puesto que se realizó con base en las relaciones entre las variables aleatorias asociadas a los proyectos y algunas de estas relaciones son mencionadas en la sección 2.8., aun 
así, no pierden generalidad ni confiabilidad. Si se revisa con detalle la matriz de comparaciones pareadas (Tabla 2), se pueden observar algunas inconsistencias. Sin embargo, esto no sólo es permitido, sino que además es necesario, ya que da flexibilidad para ingresar nueva información y así cambiar las relaciones existentes.

\subsection{Caso de estudio para la aplicación de los modelos}

Para el caso concreto se desea generar un conjunto de opciones formado por las parejas ordenadas [valor de operación, riesgo], que le permita a la organización y más específicamente a las personas encargadas, tomar una decisión de inversión.

Tabla 2. Matriz de comparaciones pareadas (índice económico)

\begin{tabular}{ccccccccccc}
\hline Proyecto & $\mathbf{1}$ & $\mathbf{2}$ & $\mathbf{3}$ & $\mathbf{4}$ & $\mathbf{5}$ & $\mathbf{6}$ & $\mathbf{7}$ & $\mathbf{8}$ & $\mathbf{9}$ & $\mathbf{1 0}$ \\
\hline $\mathbf{1}$ & 1 & $1 / 3$ & $1 / 3$ & $1 / 3$ & $1 / 5$ & $1 / 5$ & $1 / 5$ & $1 / 2$ & $1 / 3$ & $1 / 3$ \\
$\mathbf{2}$ & 3 & 1 & $1 / 2$ & $1 / 2$ & $1 / 3$ & $1 / 3$ & $1 / 3$ & $1 / 5$ & $1 / 4$ & $1 / 2$ \\
$\mathbf{3}$ & 3 & 2 & 1 & $1 / 3$ & $1 / 2$ & $1 / 2$ & $1 / 3$ & $1 / 3$ & $1 / 2$ & $1 / 3$ \\
$\mathbf{4}$ & 3 & 2 & 3 & 1 & $1 / 5$ & $1 / 3$ & $1 / 3$ & $1 / 5$ & $1 / 2$ & $1 / 3$ \\
$\mathbf{5}$ & 5 & 3 & 2 & 5 & 1 & $1 / 3$ & $1 / 2$ & $1 / 3$ & $1 / 3$ & $1 / 3$ \\
$\mathbf{6}$ & 5 & 3 & 2 & 3 & 3 & 1 & $1 / 3$ & $1 / 2$ & $1 / 2$ & $1 / 2$ \\
$\mathbf{7}$ & 5 & 3 & 3 & 3 & 2 & 3 & 1 & $1 / 3$ & $1 / 3$ & $1 / 3$ \\
$\mathbf{8}$ & 2 & 5 & 3 & 5 & 3 & 2 & 3 & 1 & $1 / 3$ & $1 / 2$ \\
$\mathbf{9}$ & 3 & 4 & 2 & 2 & 3 & 2 & 3 & 3 & 1 & $1 / 3$ \\
$\mathbf{1 0}$ & 3 & 2 & 3 & 3 & 3 & 2 & 3 & 2 & 3 & 1 \\
\hline
\end{tabular}

Tabla 3. Matriz normalizada (índice económico)

\begin{tabular}{ccccccccccc}
\hline Proyecto & $\mathbf{1}$ & $\mathbf{2}$ & $\mathbf{3}$ & $\mathbf{4}$ & $\mathbf{5}$ & $\mathbf{6}$ & $\mathbf{7}$ & $\mathbf{8}$ & $\mathbf{9}$ & $\mathbf{1 0}$ \\
\hline $\mathbf{1}$ & $1 / 33$ & $1 / 76$ & $1 / 59$ & $1 / 69$ & $1 / 81$ & $1 / 58$ & $1 / 60$ & $5 / 84$ & $4 / 85$ & $2 / 27$ \\
$\mathbf{2}$ & $1 / 11$ & $3 / 76$ & $2 / 79$ & $1 / 46$ & $1 / 49$ & $1 / 35$ & $1 / 36$ & $1 / 42$ & $3 / 85$ & $1 / 9$ \\
$\mathbf{3}$ & $1 / 11$ & $3 / 38$ & $5 / 99$ & $1 / 69$ & $2 / 65$ & $3 / 70$ & $1 / 36$ & $1 / 25$ & $6 / 85$ & $2 / 27$ \\
$\mathbf{4}$ & $1 / 11$ & $3 / 38$ & $5 / 33$ & $1 / 23$ & $1 / 81$ & $1 / 35$ & $1 / 36$ & $1 / 42$ & $6 / 85$ & $2 / 27$ \\
$\mathbf{5}$ & $5 / 33$ & $9 / 76$ & $1 / 10$ & $19 / 88$ & $4 / 65$ & $1 / 35$ & $1 / 24$ & $1 / 25$ & $4 / 85$ & $2 / 27$ \\
$\mathbf{6}$ & $5 / 33$ & $9 / 76$ & $1 / 10$ & $11 / 85$ & $17 / 92$ & $3 / 35$ & $1 / 36$ & $5 / 84$ & $6 / 85$ & $1 / 9$ \\
$\mathbf{7}$ & $5 / 33$ & $9 / 76$ & $5 / 33$ & $11 / 85$ & $9 / 73$ & $10 / 39$ & $1 / 12$ & $1 / 25$ & $4 / 85$ & $2 / 27$ \\
$\mathbf{8}$ & $2 / 33$ & $15 / 76$ & $5 / 33$ & $19 / 88$ & $17 / 92$ & $13 / 76$ & $1 / 4$ & $5 / 42$ & $4 / 85$ & $1 / 9$ \\
$\mathbf{9}$ & $1 / 11$ & $3 / 19$ & $1 / 10$ & $7 / 81$ & $17 / 92$ & $13 / 76$ & $1 / 4$ & $5 / 14$ & $12 / 85$ & $2 / 27$ \\
$\mathbf{1 0}$ & $1 / 11$ & $3 / 38$ & $5 / 33$ & $11 / 85$ & $17 / 92$ & $13 / 76$ & $1 / 4$ & $5 / 21$ & $36 / 85$ & $2 / 9$ \\
\hline
\end{tabular}

Tabla 4. Vector de ponderación (índice económico)

\begin{tabular}{cc}
\hline Proyecto & Ponderación \\
\hline $\mathbf{1}$ & $1 / 33$ \\
$\mathbf{2}$ & $2 / 47$ \\
$\mathbf{3}$ & $5 / 96$ \\
$\mathbf{4}$ & $3 / 50$ \\
$\mathbf{5}$ & $8 / 91$ \\
$\mathbf{6}$ & $8 / 77$ \\
$\mathbf{7}$ & $2 / 17$ \\
$\mathbf{8}$ & $11 / 73$ \\
$\mathbf{9}$ & $5 / 31$ \\
$\mathbf{1 0}$ & $13 / 67$ \\
\hline
\end{tabular}


Acontinuación se presentarán las principales características de los proyectos que están inscritos en el plan de inversión que tiene la empresa, éstas se constituyen en la base para definir los diferentes supuestos que se necesitan al momento de realizar las proyecciones financieras, donde se destaca la asignación de las funciones de distribución asociadas a las variables que se identificaron como críticas en el modelo de dichas proyecciones.

La organización desea realizar la selección y programación de diez proyectos para un horizonte de tiempo de cuatro años. La inversión para todos los proyectos se debe realizar en el instante cero del período de planeación y por una sola vez.

Para realizar las diferentes alternativas de negocio, la organización proyectó la demanda de la fuerza laboral y de maquinaria, como también la disponibilidad de estas, información relevante para la construcción de las restricciones técnicas de los dos modelos.

Adicional a los elementos citados, se cuenta con registros respecto a la tasa de crecimiento anual de los ingresos, el margen EBITDA, la inversión inicial, el incremento anual en capital de trabajo para cada uno de los proyectos en valoración, la tasa de impuestos, el capital disponible para invertir y el costo de capital sobre el cual se realizaría la evaluación. Parte de esta información se muestra en las Tablas 5 y 6.

Tabla 5. Inversión inicial por proyecto

\begin{tabular}{cc}
\hline Proyecto & $\begin{array}{c}\text { Inversión } \\
\text { Inicial }\end{array}$ \\
\hline $\mathbf{1}$ & 1,830 \\
$\mathbf{2}$ & 1,890 \\
$\mathbf{3}$ & 2,380 \\
$\mathbf{4}$ & 2,140 \\
$\mathbf{5}$ & 2,370 \\
$\mathbf{6}$ & 1,590 \\
$\mathbf{7}$ & 2,500 \\
$\mathbf{8}$ & 2,050 \\
$\mathbf{9}$ & 1,980 \\
$\mathbf{1 0}$ & 1,700 \\
\hline
\end{tabular}

Tabla 6. Parámetros relevantes

\begin{tabular}{cccc}
\hline $\begin{array}{c}\text { Capital } \\
\text { disponible }\end{array}$ & $\begin{array}{c}\text { Impuesto, } \\
\%\end{array}$ & $\begin{array}{c}\text { Horizonte } \\
\text { de } \\
\text { planeación }\end{array}$ & $\begin{array}{c}\text { Costo de } \\
\text { capital, } \\
\%\end{array}$ \\
\hline 12000 & 30 & 4 & 14 \\
\hline
\end{tabular}

Además, se consideraron las siguientes condiciones especiales para la realización de los proyectos:

- La realización del proyecto 8 implica la ejecución del proyecto 4 y viceversa.

- La realización del proyecto 3 implica la ejecución del proyecto $10 \mathrm{y}$ viceversa.

- La realización del proyecto 6 implica la ejecución del proyecto 3 y viceversa.

- La realización del proyecto 5 implica la ejecución del proyecto 1, pero la realización del proyecto 1 no implica la ejecución del proyecto 5.

- La realización del proyecto 3 implica la ejecución del proyecto 2 , pero la realización del proyecto 2 no implica la ejecución del proyecto 3.

\section{Resultados y Discusión}

Posterior a la implementación y ejecución del modelo computacional asociado a cada uno de los dos modelos propuestos, se realizó un análisis de los resultados asociados a los criterios de evaluación, los cuales fueron obtenidos por la aplicación del AHP. Es importante anotar que éstos sólo son necesarios para el modelo MG. Así mismo se estudiaron los resultados de los VPN de los proyectos y su respectivo análisis de sensibilidad, la estimación del riesgo individual, tomando como base la forma tradicional de estimarlo (probabilidad $\mathrm{VPN}<0$ ), finalmente se analizaron los resultados obtenidos para conformar el portafolio óptimo, en cada uno de los modelos y su comparación en dos escenarios 


\subsection{Resultados de la aplicación del AHP}

Como se explicó en la sección 2.7., siguiendo los pasos descritos y necesarios para la aplicación de AHP para cada criterio evaluado, bajo una hoja electrónica de Excel, se lograron obtener las ponderaciones de los índices de valoración para cada uno de los proyectos, estos resultados se condensan en la Tabla 7. En ella se puede observar que existen proyectos muy atractivos puesto que sus ponderaciones así lo muestran. Sin embargo, sería apresurado pensar que pueden formar parte del portafolio final, de igual forma no se pueden desechar otros que no poseen buenas ponderaciones.

Tabla 7. Pesos de los índices obtenidos por AHP para los 10 proyectos

\begin{tabular}{cccc}
\hline \multirow{2}{*}{ Proyecto } & \multicolumn{3}{c}{ Índice } \\
\cline { 2 - 4 } & $\begin{array}{c}\text { Económico, } \\
\mathbf{\%}\end{array}$ & $\begin{array}{c}\text { Financiero, } \\
\mathbf{\%}\end{array}$ & $\begin{array}{c}\text { Social, } \\
\mathbf{\%}\end{array}$ \\
\hline 1 & 3.01 & 2.68 & 3.66 \\
2 & 4.24 & 4.98 & 4.73 \\
3 & 5.20 & 5.23 & 6.05 \\
4 & 6.01 & 7.08 & 6.89 \\
5 & 8.79 & 7.61 & 7.59 \\
6 & 10.39 & 9.59 & 9.93 \\
7 & 11.74 & 12.38 & 12.7 \\
8 & 15.07 & 13.33 & 13.86 \\
9 & 16.13 & 19.37 & 16.06 \\
10 & 19.40 & 17.78 & 18.46 \\
\hline
\end{tabular}

\subsection{Resultados de los valores presentes netos}

Para la implementación de ambos modelos era necesario conocer los valores presentes netos de los proyectos (VPN). Por lo tanto, se calcularon los flujos de caja netos (FCN) de los proyectos involucrando las variables aleatorias (crecimiento de los ingresos, margen EBITDA e incremento en el capital de trabajo) con sus respectivas distribuciones de probabilidad, las cuales se mencionaron en las secciones 2.7 y 2.8. La Tabla 8 corresponde a los resultados obtenidos para el FCN del proyecto dos, con un nivel de seguridad del $95 \%$ y 10000 iteraciones. De igual forma se obtuvieron los FCN de los otros proyectos.

Una vez calculados los FCN fue posible el cálculo de los VPN de los proyectos. Los resultados más significativos obtenidos en la simulación se observan en la Tabla 9, evaluando como variable de salida el VPN de los proyectos. En ellos se puede observar que la variabilidad de los VPN es significativa. La razón fundamental para que esto ocurra es la influencia que tienen las tres variables aleatorias involucradas en este cálculo, por consiguiente el número de combinaciones posibles, para generar variabilidad es bastante extenso.

Desde el punto de vista estadístico, los coeficientes de variabilidad de los VPN para los proyectos cinco y nueve son muy altos $(-136.23 \mathrm{y}-$ 456.10 respectivamente), esto se debe al valor del VPN cercano a 0 .

Tabla 8. Cálculo de los FCN para el proyecto 2

\begin{tabular}{ccccccccc}
\hline Año & Ingresos & EBITDA $^{\mathbf{1}}$ & $\mathbf{U O}^{\mathbf{2}}$ & $\mathbf{I m p}^{\mathbf{3}}$ & UODI $^{\mathbf{4}}$ & Capex $^{\mathbf{5}}$ & $\mathbf{W C}^{\mathbf{6}}$ & $\mathbf{F C N}^{\mathbf{7}}$ \\
\hline & & & & & & & & - \\
0 & & & & & & 1,890 & & 1,890 \\
& 1,600 & 640 & 168 & 50 & 117 & & 205 & 502 \\
2 & 1,744 & 698 & 225 & 68 & 158 & & 223 & 564 \\
3 & 1,901 & 760 & 288 & 86 & 202 & & 243 & 632 \\
4 & 2,072 & 829 & 356 & 107 & 249 & & 265 & 706 \\
\hline
\end{tabular}

\footnotetext{
EBITDA1: Earnings before interest, taxes, depreciation and amortization (Utilidad antes de intereses impuestos depreciaciones y amortizaciones) UO2: Utilidad operacional

Imp3: Impuestos

UODI4: Utilidad operacional después de impuestos

Capex5: Capital expenditures

WC6: Working capital variation

FCN7: Flujo de caja neto
} 
Tabla 9. Resultados del VPN para los diez proyectos

\begin{tabular}{lcccccccccc}
\hline Estadisticas & $\mathbf{1}$ & $\mathbf{2}$ & $\mathbf{3}$ & $\mathbf{4}$ & $\mathbf{5}$ & $\mathbf{6}$ & $\mathbf{7}$ & $\mathbf{8}$ & $\mathbf{9}$ & $\mathbf{1 0}$ \\
\hline Iteraciones & 10,000 & 10,000 & 10,000 & 10,000 & 10,000 & 10,000 & 10,000 & 10,000 & 10,000 & 10,000 \\
Media & 109 & -20 & -46 & 169 & -1 & 75 & 97 & 144 & 0 & 75 \\
$\begin{array}{l}\text { Desviación } \\
\text { Estandar }\end{array}$ & 101 & 88 & 135 & 127 & 122 & 51 & 72 & 104 & 72 & 95 \\
$\begin{array}{l}\text { Varianza } \\
\begin{array}{l}\text { Coeficiente } \\
\text { de }\end{array}\end{array}$ & 10,157 & 7,758 & 18,347 & 16,059 & 14,773 & 2,589 & 5,209 & 10,801 & 5,158 & 8,946 \\
$\begin{array}{l}\text { Variabilidad } \\
\text { Mínimo }\end{array}$ & 0.92 & -4.36 & -295 & 0.75 & -136.23 & 0.68 & 0.74 & 0.72 & -456.1 & 1.26 \\
Máximo & -191 & -296 & -417 & -234 & -340 & -95 & -139 & -201 & -212 & -242 \\
\hline & 452 & 306 & 394 & 538 & 347 & 219 & 346 & 480 & 279 & 361 \\
\hline
\end{tabular}

Con respecto a los VPN obtenidos para los proyectos dos, tres, cinco y nueve no serían factibles de realizar. Sin embargo, la evaluación individual no es un factor preponderante de descarte o inclusión de un proyecto cuando se están realizando portafolios de inversión. En cuanto a la sensibilidad, las Figuras 3 y 4 muestran como la variable de mayor contribución a los resultados finales de los VPN corresponde al margen EBITDA.

3.3. Análisis de riesgo individual de los proyectos simulados

Este análisis corresponde a la estimación de la probabilidad de no factibilidad del proyecto a realizar. La Figura 5 ilustra el porcentaje de riesgo asociado al proyecto 4 , de igual forma se obtuvieron los riesgos individuales asociados a cada uno de los proyectos cuyos resultados se condensan en la Tabla 10. Allí se puede observar como el riesgo individual asociado a los proyectos dos, tres y nueve es de 58\%, 49\% y 51\%.

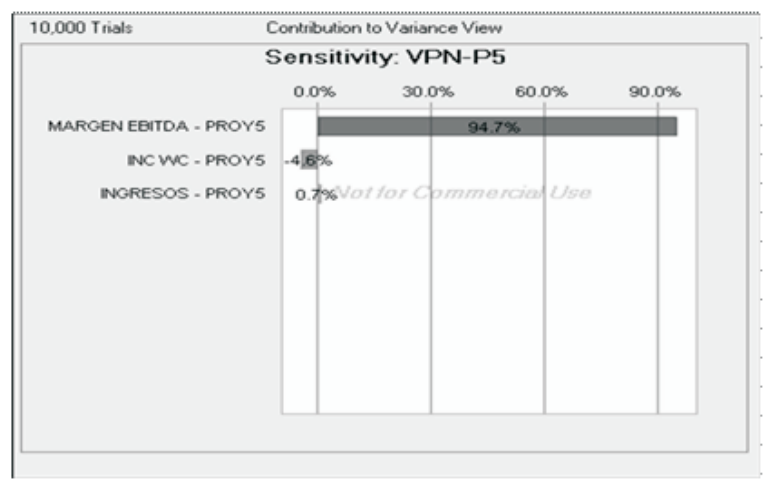

Figura 3. Contribución a la varianza para la simulación del $V P N$ del proyecto 5

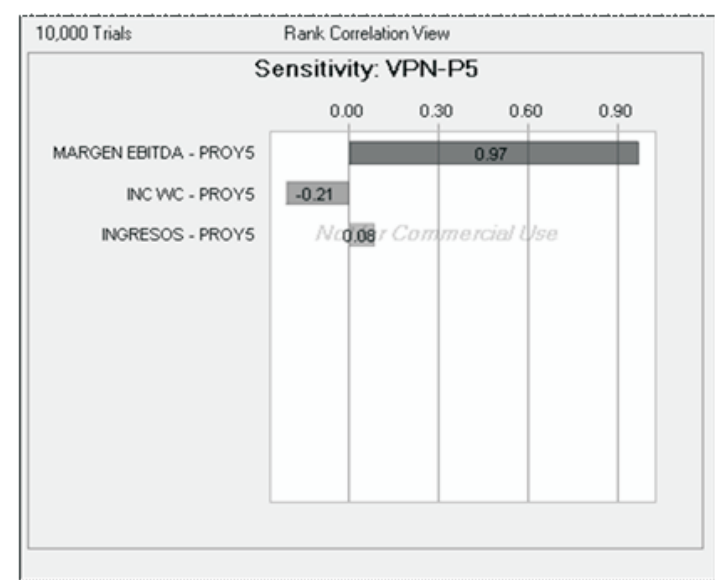

Figura 4. Correlación para la simulación del VPN del proyecto 5 




Figura 5. Riesgo asociado al proyecto 4

Tabla 10. Análisis de riesgo individual

\begin{tabular}{ccc}
\hline Proyecto & $\begin{array}{c}\text { P(VPN<0) } \\
(\%)\end{array}$ & VPN \\
\hline 1 & 13.79 & 109 \\
2 & 58.48 & -20 \\
3 & 65.00 & -46 \\
4 & 10.88 & 169 \\
5 & 50.9 & -1 \\
6 & 7.77 & 75 \\
7 & 9.16 & 97 \\
8 & 9.64 & 144 \\
9 & 49.51 & 0 \\
10 & 21.53 & 75 \\
\hline
\end{tabular}

\subsection{Resultados de la aplicación del modelo MG}

Con base en la información recolectada, tanto la suministrada por la organización, como la obtenida para los FCN, los VPN de los proyectos, el cálculo de los vectores de ponderación de los tres índices evaluados y demás detalles necesarios para la implementación del modelo computacional en la hoja de cálculo, se realizó la simulación con el optimizador Opquest de Crystal Ball, ${ }^{\circledR}$ con el fin determinar el portafolio óptimo. Los resultados obtenidos se presentan en la Figura 6.

Los resultados de la optimización del modelo MG indican que en función de obtener el mejor portafolio de inversión, es necesario la realización de los proyectos cuatro, cinco, siete, ocho y nueve
Este resultado confirma lo expuesto en los análisis del VPN y del riesgo individual, ya que los proyectos cinco y nueve tienen un VPN cercano a cero, de igual forma el riesgo individual asociado a estos dos proyectos oscila entre el $49 \%$ y $51 \%$ (Tabla 10), y aún así forman parte del portafolio óptimo. La razón de este hecho, es que el proyecto nueve tiene la mejor ponderación para el índice financiero y ocupa el segundo lugar en el índice social como lo muestra la Tabla 7 (condensa los resultados del AHP), el consumo de recursos (restricciones técnicas horas-hombre y horas máquina) es bastante aceptable, puesto que son bajos. Con respecto al proyecto cinco, se observa un menor consumo de recursos, aún cuando no goza de las mejores ponderaciones en cuanto a los índices evaluados.

La selección de los proyectos cuatro y ocho es razonable, por cuánto sus indicadores son adecuados, pero adicional a ello existe una restricción de precedencia bidireccional, lo cual los convierte en firmes candidatos a formar parte del portafolio en cualquier escenario.

Los anteriores análisis llevan a la formulación de la pregunta ¿cuál es la razón para no seleccionar proyectos atractivos como el seis y el diez?, donde quizás, éste último parece ser el candidato más fuerte para pertenecer al portafolio óptimo. La respuesta a la pregunta se fundamenta en el hecho de que en el modelo existen restricciones de precedencia, las cuales se encuentran en el caso de estudio. La selección del proyecto seis o del proyecto diez, implica la ejecución del proyecto 


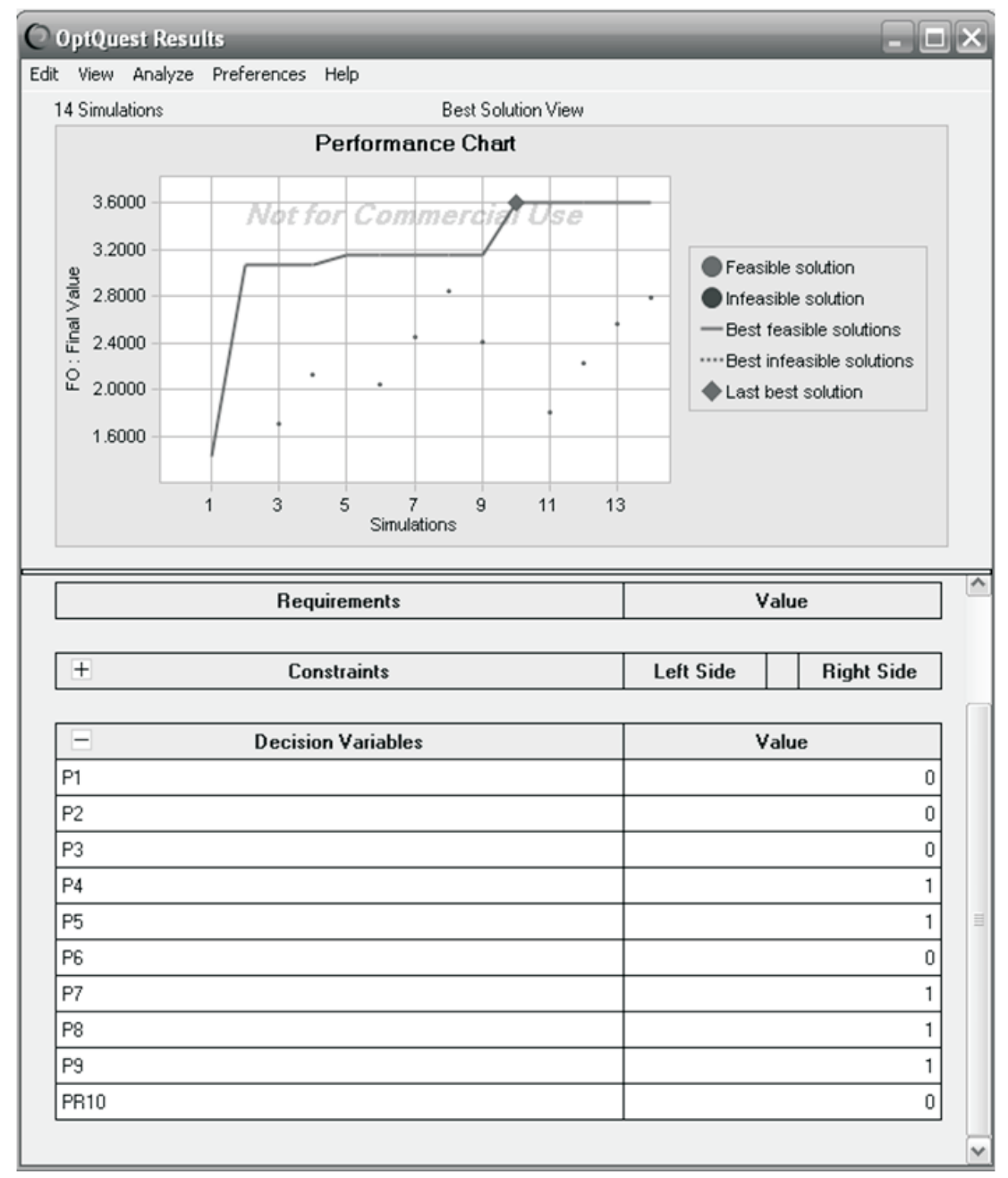

Figura 6. Resultados de la optimización del modelo $M G$

tres, el cual presenta indicadores muy deficientes.

Con relación a la selección del proyecto siete, sus números son aceptables y no tiene relaciones de precedencia que impidan su realización.

\subsection{Resultados de la aplicación del modelo MS}

Para este modelo no era necesario calcular los pesos para los indicadores económico, financiero y social, puesto que las dos funciones objetivo están asociadas al VPN de los proyectos y al riesgo medido por la semivarianza, respectivamente. Los resultados obtenidos se muestran en la Figura 7. Allí se pueden observar los resultados de la optimización del modelo MS que indican que el mejor portafolio de inversión se obtiene al ejecutar los proyectos uno, cuatro, cinco, siete y ocho. La selección de los proyectos ocho, cuatro y siete, y la exclusión de los proyectos seis y diez, tiene la misma explicación que se proporcionó para el modelo MG en la anterior sección. Sin embargo, es preciso aclarar que la selección del proyecto cinco no es la misma; la inclusión de este proyecto en el portafolio óptimo se debe a la relación de precedencia que tiene con el proyecto uno, pues la realización del proyecto uno implica la ejecución del proyecto cinco. Los indicadores del proyecto uno lo convierten en un candidato bastante atractivo para seleccionarse por el modelo MS, puesto que tiene un VPN, alto, un riesgo manejable (Tabla 10) y un consumo de recursos bajo, además el modelo no involucra los índices del modelo MG. 


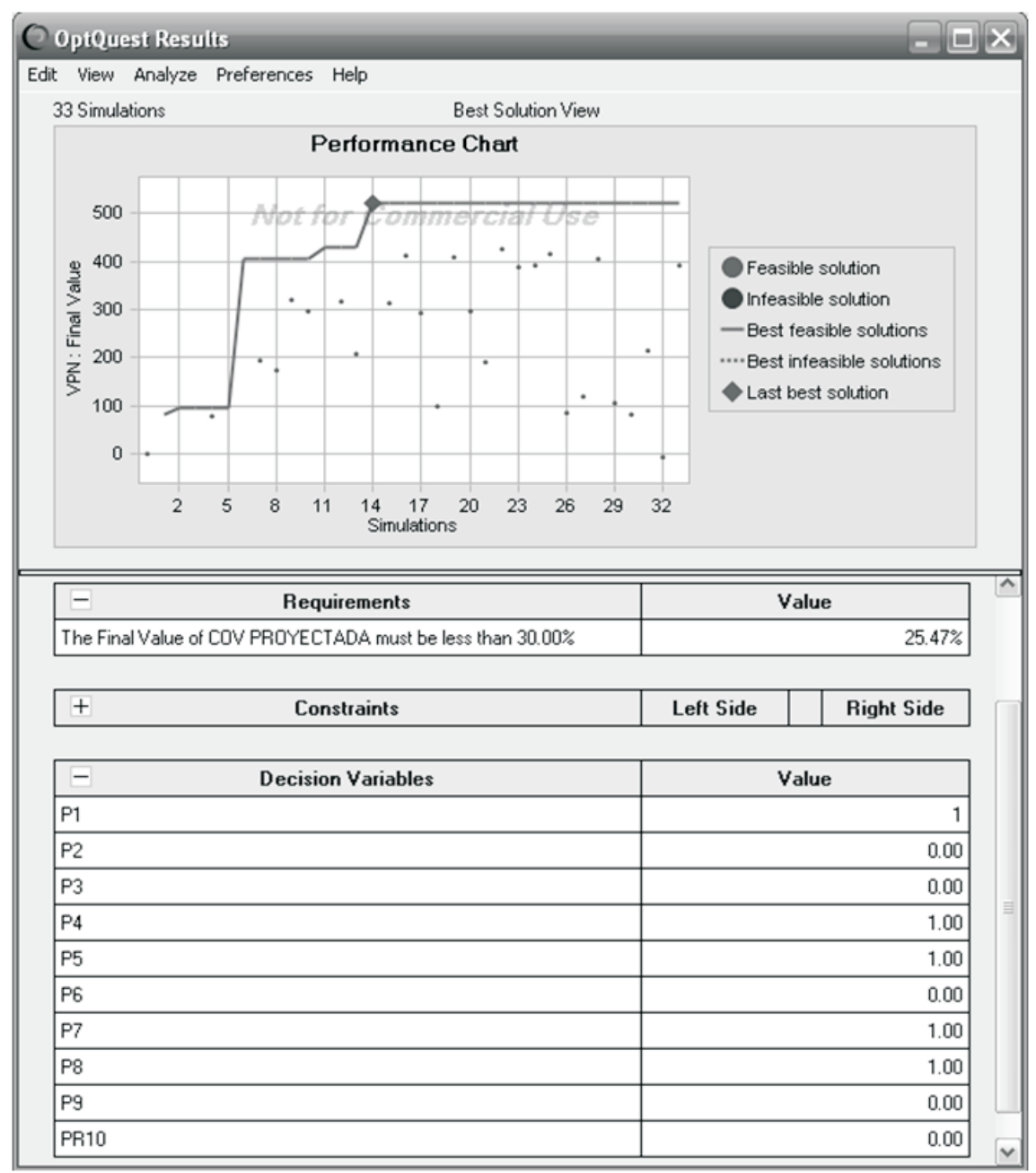

Figura 7. Resultados de la optimización para el modelo MS

\subsection{Análisis de escenarios}

Con el objetivo de ponderar mejor los alcances que pueden proporcionar los modelos, se realiza un análisis de escenarios cuyos principales elementos se consideran a continuación.

\subsubsection{Incremento en la disponibilidad de capital}

El primer escenario consiste en incrementar la disponibilidad de capital a invertir, la Tabla 11 condensa los resultados de la simulación. Con base en ella se observa que ambos modelos forman el mismo portafolio. Este es un hecho bastante lógico, que se podría esperar, puesto que el aumento que se hizo fue direccionado, con el ánimo de que a lo sumo se incrementara en uno la cantidad de proyectos. De los anteriores análisis sabemos que los proyectos dos y tres presentan los peores indicadores, por lo tanto, difícilmente se incluirían bajo este cambio, además del hecho de que no incluir el proyecto tres lleva implícitamente a la no inclusión de los proyectos seis y diez. Motivo por el cual el modelo MG adhiere a su portafolio, el proyecto menos perjudicial para sus intereses (proyecto 1). Lo mismo hace el modelo MS que en su caso agrega el proyecto nueve.

Desde luego el hecho de seleccionar los mismos proyectos, lleva consigo la obtención de resultados iguales en su VPN, no así en el riesgo, puesto que la forma de evaluarlo es diferente. 
Tabla 11. Resultados del escenario Incremento de Capital

\begin{tabular}{ccc}
\hline Proyecto & Modelo MG & Modelo MS \\
\hline 1 & $*$ & $*$ \\
3 & & \\
4 & $*$ & $*$ \\
5 & $*$ & $*$ \\
6 & $*$ & $*$ \\
7 & $*$ & $*$ \\
8 & $*$ & \\
9 & & 25.94 \\
10 & 28.51 & \\
Riesgo del & & 12870 \\
Portafolio, \% & 12870 & \\
Inversión \\
total
\end{tabular}

Tabla 12. Resultados del escenario Variación del Costo de Capital

\begin{tabular}{|c|c|c|c|c|c|c|}
\hline \multirow{4}{*}{ Proyecto } & \multicolumn{6}{|c|}{ Costo de capital } \\
\hline & \multicolumn{3}{|c|}{ Modelo MG } & \multicolumn{3}{|c|}{ Modelo MS } \\
\hline & $8 \%$ & $14 \%$ & $35 \%$ & $8 \%$ & $14 \%$ & $35 \%$ \\
\hline & \multicolumn{6}{|c|}{ Proyectos seleccionados } \\
\hline 1 & & & & $*$ & $*$ & \\
\hline 2 & & & & & & $*$ \\
\hline \multicolumn{7}{|l|}{3} \\
\hline 4 & $*$ & $*$ & $*$ & $*$ & * & $*$ \\
\hline 5 & * & $*$ & $*$ & $*$ & * & \\
\hline \multicolumn{7}{|l|}{6} \\
\hline 7 & $*$ & $*$ & $*$ & $*$ & $*$ & \\
\hline 8 & $*$ & $*$ & $*$ & * & $*$ & $*$ \\
\hline 9 & * & $*$ & $*$ & & & \\
\hline \multicolumn{7}{|l|}{10} \\
\hline Riesgo del & & & & & & \\
\hline $\begin{array}{c}\text { Portafolio, } \\
\%\end{array}$ & 28.53 & 28.51 & 28.49 & 25.38 & 28.21 & 23.45 \\
\hline $\begin{array}{c}\text { Inversión } \\
\text { total }\end{array}$ & 11040 & 11040 & 11040 & 11040 & 11040 & 6080 \\
\hline VPN total & 2111 & 409 & -3601 & 2219 & 518 & -1845 \\
\hline
\end{tabular}

\subsubsection{Variación del Costo de Capital}

El hecho más relevante lo constituye la insensibilidad del modelo MG ante los cambios de este parámetro. Como se puede apreciar en la Tabla 12, el modelo conduce a la misma selección, por tanto los resultados obtenidos son los mismos, 
en cuanto a inversión y el riesgo asociado al portafolio. Con respecto al VPN es sabido que depende del costo de capital y una variación de este produce un movimiento en el resultado final de dicho VPN.

En torno al riesgo es meritorio hacer un comentario especial. Es necesario recordar que el coeficiente de Gini mide la desigualdad que existe entre los proyectos, al variar el costo de capital, todos los proyectos sufren un cambio de igual magnitud, por tanto la desigualdad existente es la misma.

Esta es quizás la razón más importante para utilizar el modelo MG en la selección de proyectos de inversión, puesto que su insensibilidad ante perturbaciones ocasionales le dan un alto grado de confiabilidad en la utilización del estimador de riesgo, además, su función objetivo involucra el índice de rentabilidad y no propiamente el VPN y el riesgo.

Con respecto a los resultados obtenidos para el modelo MS y sus dos funciones objetivo, maximizar el VPN y minimizar el riesgo, en la Tabla 12 se observa que con un costo de capital de $8 \%$ se seleccionaron los mismos proyectos que con el $14 \%$ (costo de capital estimado para la empresa). La formación del portafolio es lógica, puesto que al disminuir o al aumentar la tasa de descuento, el valor de los VPN se incrementa en el primer caso y se disminuye en el segundo, esta variación lleva como resultado, una mayor acentuación de las diferencias entre estos valores.

Para ilustrar lo anterior tomamos como ejemplo los proyectos seis y diez, los cuales con base en la Tabla 10 tienen el mismo VPN (75). En realidad su diferencia está en el orden de las centésimas en este caso el costo de capital es el suministrado por la empresa $14 \%$, al realizar la simulación con una tasa de descuento del $8 \%$ el VPN del proyecto seis es de 320.7 mientras que el VPN del proyecto es de 331.2 , la diferencia ya empieza a ser significativa.

Por la misma razón, en el caso de aumentar el costo de capital a un valor del $35 \%$, proyectos como el tres y el cinco se vuelven intratables, básicamente ésta es una de las razones por las cuales el optimizador sólo escoge tres proyectos, en realidad los menos perjudiciales debido a que el modelo cuenta con esa restricción (mínimo tres). Si se omite esta restricción el optimizador no encuentra solución.

Para finalizar el análisis de este escenario es interesante observar lo que pasa con el riesgo estimado por la semivarianza en el modelo MS. Como se puede ver en la Tabla 12, la variación no es significativa. En realidad esto se debe a la forma de medir el riesgo con este método, puesto que el toma como referencia el VPN, e involucra en sus cálculos sólo los valores que están por debajo de él, por lo tanto independiente del valor del costo de capital con el que se efectúen los cálculos, la variación del VPN solo es posicional, pero la cantidad de valores que se encuentran por debajo de él, es prácticamente la misma, y consecuentemente las variaciones del valor en riesgo son relativamente insignificantes.

\section{Conclusiones}

La selección de proyectos de inversión es una tarea compleja. Sin embargo los modelos matemáticos aún cuando no toman la decisión, sí se convierten en elementos de relevante importancia para apoyar el proceso de toma de decisiones, debido a que entregan herramientas que permiten ampliar su panorama, con mejores juicios valorativos en procura de tomar la decisión más acertada, en el corto, mediano y largo plazo.

Antes del planteamiento de un modelo matemático para la selección de un portafolio de inversión, se deben definir los conceptos de rentabilidad y riesgo con el fin de proceder a su análisis y medición, puesto que de alguna forma son los parámetros más importantes al momento de la conformación del portafolio. Sin embargo, la mayor dificultad no radica en la definición de estos conceptos, sino en el método de estimación que debe utilizarse para su obtención.

Evaluar individualmente un proyecto $\mathrm{y}$ descartarlo por presentar un valor alto en su riesgo asociado o un valor bajo en su rentabilidad, no es una técnica efectiva para conformar un portafolio de inversión, ya que la presencia de otros 
proyectos dentro del mismo puede mitigar o disminuir los efectos negativos de estos proyectos considerados no factibles. La presente investigación corrobora este hecho. En el análisis de resultados se muestra, como los dos modelos propuestos seleccionan un proyecto con un nivel de riesgo alto, si a esto se le agrega, la inserción de otros indicadores en la evaluación de los proyectos, éstos pueden llegar a ser más importantes que el de la rentabilidad, más aun cuando forman parte de la función objetivo como lo es en este caso para el modelo MG.

Lo anterior implica la necesidad de establecer por parte de las organizaciones, verdaderas herramientas de valoración y ordenamiento de proyectos, que se adecuen a sus dinámicas internas. Durante el proceso de elaboración de esta metodología, se dio a conocer la evolución que se ha dado alrededor de las diferentes técnicas para valorar portafolios de inversión que implican alta incertidumbre, además de resaltar el valor de la flexibilidad de tomar decisiones por parte de los directores de los proyectos, como respuesta al medio en que se desarrollan éstos, que es incierto, debido a la variación continua propia de nuestra época. Los modelos desarrollados permiten establecer las diferencias cuando se evalúa el impacto de variables diferentes a las tradicionales de los proyectos sobre la estrategia de selección y programación de planes de inversión.

Existen muchas y buenas herramientas para la obtención de un portafolio óptimo pero no han tenido buena difusión a nivel empresarial, debido a la dificultad que ocasiona la determinación de las variables y su incertidumbre durante el proceso de valoración. En la medida que se construyan modelos de tipo estocásticos que reflejen más de cerca la realidad y al tiempo sean más amigables, será más frecuente su uso, ya que pueden contar con un amplio número de escenarios para evaluar en corto tiempo y a bajo costo.

Con respecto a la estimación del riesgo de un portafolio de inversión la medida más conocida y ampliamente difundida es la varianza, la cual mide la volatilidad de los retornos alrededor de la media. Es preciso recordar que el vector de medias muestrales y la matriz de covarianzas muestrales son estadísticos suficientes para distribuciones normales conjuntas. Sin embargo, la percepción sobre el riesgo de una inversión está generalmente asociada a las variaciones de los rendimientos por debajo de una tasa de retorno mínima. Este tipo de riesgo puede estimarse mediante otros estadísticos que tengan la ventaja de representar una medida más consistente con la noción común de riesgo y que además pueda utilizarse en cualquier tipo de distribución asociada a los retornos independiente de su simetría, esta es quizás la mayor razón por la cual en la presente investigación se utiliza el coeficiente de Gini como estimador de riesgo en el primer modelo y la semivarianza en el segundo. Los cuales por su forma de valorar el riesgo producen un alto grado de confiabilidad en su utilización, debido a la insensibilidad que tienen frente a perturbaciones ocasionales que se puedan presentar en algunos parámetros.

De otro lado, es importante resaltar que el hecho de contar actualmente con procesadores tan veloces y paquetes como Opquest, es una ventaja significativa, puesto que se simplifica el trabajo de manera sustancial. En el caso particular del presente trabajo fue muy bondadoso, especialmente en el modelo MS, ya que el hecho de tener una función bi-objetivo hace complejo su diseño computacional, sin embargo y aun cuando el optimizador solicita la priorización de uno de los objetivos, no importa a cual se le asigna la prioridad, ya que el algoritmo que realiza se encarga de encontrar la frontera eficiente y de allí toma la mejor decisión, la única diferencia que presenta es en el número de simulaciones que tiene que realizar para su hallazgo.

Hasta el momento de culminación de esta investigación, no se conoce un método universal que se pueda aplicar a la gran variedad de situaciones alusivas a la selección y priorización de proyectos, puesto que si bien se pueden organizar por grupos, con características similares, existen algunas particularidades dentro de los problemas que no pueden resolverse por todos los métodos y en algunos casos sólo pueden resolverse de forma parcial.

Por lo anterior la presente investigación con seguridad sirve como base para realizar estudios 
más elaborados. Particularmente es importante integrar condiciones dentro de estos modelos de optimización, de tal forma que éstos tengan la capacidad de evaluar situaciones como: la variabilidad en los tiempos de inversión inicial y final, es decir involucrar las ventanas de tiempo para la apertura y cierre de los proyectos; incluir el análisis presupuestal período a período; entre otras características, que permitan obtener un modelo más dinámico y en lo posible más acorde con las realidades de las organizaciones.

\section{Referencias Bibliográficas}

Azarang, M.,\& Garcia, E. (1998) Simulación y análisis de modelos estocásticos. Segunda Edición. McGraw Hill.

Branke, J., Scheckenbach, M., Stein., \& M; Schmeck, H. (2008). Portfolio optimization with an envelope-based multiobjective evolutionary algorithm. European Journal of Operational Research, 684-693.

Enea, M. \& Piazza, T. (2004). Project selection by constrained fuzzy AHP. Fuzzy Optimization and Decision Making 3, 39-62.

Estrada, J. IESE (Business School. Universidad de Navarra).(2003). Mean-semivariance behavior (II). http// www.iese.edu.es/research/pdfs/DI0493-E.pdf

Fernandez, A. \& Gómez, S. (2007). Portfolio selection using neural networks.Computers \& Operations Research 34, 1177-1191.

Ghorbani, S \& Rabbani, M (2009). A new multiobjective algorithm for a project selection problem. Advances in Engineering Software 40, 914

Huang, X. (2007a). Optimal project selection with random fuzzy parameters. International Journal Production Economics 106, 513-522.
Huang, X. (2007b). Two new models for portfolio selection with stochastic returns taking fuzzy information. European Journal of Operational Research 180, 396-405.

Huang, X. (2008). Risk curve and fuzzy portfolio selection. Computers and Mathematics with Applications 55, 1102-1112.

Law, A. \& Kelton, D. Simulation modeling and analysis. Tercera Edición. Mc Graw Hill. 2000.

Lin, C., Tan, B., \& Hsieh P. (2005). Aplication of the fuzzy weighted average in strategic portfolio management. Decision Sciences 36 (3)

Marantes, L., Medaglia, A., \& Palacios, F. (2005). Aproximación con algoritmos evolutivos para el problema de selección de proyectos con restricciones lineales y variables binarias bajo ambientes de incertidumbre.

http://elavio2005.uniandes.edu.co

Markowitz, H. (1952). Portfolio selection. The Journal of Finance 7 (1), 77-91.

Medaglia, A., Graves, S., \& Ringuest, J. (2007). A multiobjective evolutionary approach for linearly constrained project selection under uncertainty. European Journal of Operational Research 179, 869-894.

Medaglia, A., Hueth, D., Mendieta, J., \& Sefair, J. (2008). A multiobjective model for the selection and timing of public enterprise projects. SocioEconomic Planning Sciences 42, 3-45

Ringuest, J., Graves, S., \& Case, R. (2004). MeanGini analysis in R\&D portfolio selection. European Journal of Operational Research 154, 157-169.

Sefair, J., Mendez, C., \& Medaglia, A. (2008). Mean-semivariance model for project selection: an illustration from the oil and gas industry. $\mathrm{http} / / /$ dspace.uniandes.edu.co.

Shalit, H., \& S. Yitzhaki (1984) Mean-Gini, Portfolio Theory, and the Pricing of Risky Assets, Journal of Finance 39 (5), 1449-68 
Sitaram, M., Subramanian, S., \& Rao, U. (2004).Usefulness of genetic algorithm model for dynamic portfolio selection. Journal of Financial Management \& Analysis. 17 (1), 45-53.

Tiryaki, F., \& Ahlatcioglu, B. (2009) Fuzzy portfolio selection using fuzzy analytic hierarchy process. Information Sciences International Journal. 179, 53-69 